\title{
Comparison of Adomian Decomposition Method and Taylor Matrix Method in Solving Different Kinds of Partial Differential Equations
}

\author{
Sinan Deniz and Necdet Bildik
}

\begin{abstract}
In this paper, we will present a comparison between the Adomian Decomposition Method (ADM) and Taylor Matrix Method by solving some well-known partial differential equations (PDEs). In order to illustrate the analysis we examined the Telegraph equation, which is considered one of the most significant partial differential equations, describe wave propagation of electric signals in a cable transmission line and Klein-Gordon equation which is encountered in several applied physics fields such as, quantum field theory , fluid dynamics, optoelectronic devices design and numerical analysis. Our study shows that the decomposition method is faster and easy to use from a computational viewpoint.
\end{abstract}

Index Terms-Adomian decomposition method, Klein gordon equation, taylor matrix method, telegraph equation.

\section{INTRODUCTION}

Many scientific and technological problems in natural and engineering sciences are modeled mathematically by partial differential equations. In physics for instance, the heat flow and the wave propagation phenomena are well defined by partial differential equations. The dispersion of a chemically reactive material is characterized by partial differential equations. In addition, most physical phenomena of fluid dynamics, quantum mechanics, electricity, plasma physics, propagation of shallow water waves, chemical reaction-diffusion and many other models are governed by partial differential equations. Besides that a substantial amount of work has been investigated for solving such models [1]-[4].

Most realistic partial differential equations do not have exact analytic solutions, so numerical techniques must be used to handle with them. Recently, several techniques including the method of Riemann invariants [5], periodic multi-grid wave form [6], variational iteration [7] and homotopy perturbation [8] have been used for the solutions of such problems. If any exact solutions exist, these numerical methods give generally approximate solutions which converge rapidly to these accurate solutions easily. The investigation of exact or approximate solutions to these partial differential equations will help us to understand phenomenas behind those models better.

The Klein-Gordon [9], [10] equation, which is derived

Manuscript received March 12, 2014; revised June 20, 2014. This work was supported in part by Celal Bayar University.

S. Deniz and N. Bildik are with the Mathematics Dept., Celal Bayar University, Manisa, Turkey (e-mail: \{sinan.deniz, necdet.bildik\}@cbu.edu.tr) from the relativistic energy formula, is one of the most important mathematical models in quantum field theory. It is a different version of the Schrödinger equation which is also one of the well known PDEs. In general, Klein-Gordon equation is used to describe dispersive wave phenomena in relativistic physics. It has attracted much attention in studying solitons and condensed matter physics, in investigating the interaction of solitons in a collisionless plasma and in examining the linear and nonlinear wave equations [11], [12]. Approximate analytical solutions of Klein-Gordon equations via the differential transform method and homotopy perturbation method were presented in [13], [14].

The linear Klein-Gordon equation in its standard form is given by

$$
u_{t t}-u_{x x}+a u=g(x, t)
$$

Subject to initial conditions

$$
u(x, 0)=f(x), u_{t}(x, 0)=h(x)
$$

where $u$ is a function of $x$ and $t, a$ is a constant and $g(x, t)$ is the source term.

Telegraph equation is also considered one of the most significant PDEs which describe wave propagation of electric signals in a cable transmission line. Many researchers have used various numerical and analytical methods to solve the telegraph equation. Mohebbi and Dehaghan [15], studied high order compact solution to solve the telegraph equation. Saadatmandi and Dehaghan [16], developed a numerical solution based on Chebyshev Tau method. Yousefi [17] used Legendre multi wavelet Galerkin method for solving the hyperbolic telegraph equation. Parkes et al. [18] used the Jacobi elliptic function expansion method, and proposed double periodic solutions.

The standard form of the telegraph equation is given by

$$
u_{x x}=a u_{t t}+b u_{t}+c u
$$

where $u(x, t)$ can be voltage or current through the wire at position $x$ and time $t$. And also we have constans $a=L C, b=G L+R C$ and $c=G R$ where $G$ is conductance of resistor, $R$ is resistance of resistor, $L$ is inductance of coil and $C$ is capacitance of capacitor.

In this paper we give short definitions of ADM and Taylor 
matrix method and we compare the performance of these methods by solving Telegraph and Klein-Gordon equation. Both methods are very effective and provide the solution in an infinite series, but ADM eliminates cumbersome computations which are needed by Taylor matrix method.

\section{Adomian Decomposition Method (ADM)}

The topic of the Adomian decomposition method has been rapidly growing in recent years. It was first proposed by George Adomian [19]. In this method the solution of functional equations is considered as the sum of an infinite series usually converging to the solution. A lot of research works have been conducted recently in applying this method to a class of linear and nonlinear partial differential equations [20]-[22]. The method is well suited to physical problems since it makes unnecessary restrictive methods and assumptions such as the linearization, perturbations etc. which may change the problem seriously.

Here we just consider the basic points of this method, further details can be found in [23].

We first consider the equation

$$
L u+R u+N u=g(t), u(0)=a
$$

where $L$ is the first order derivative which is easily invertible, $R$ is a linear differential operator and $N u$ represents the nonlinear terms and $g(t)$ is the source term.

Unknown function $u(t)$ is represented as an infinite series:

$$
u(t)=\sum_{n=0}^{\infty} u_{n}(t)
$$

And the nonlinear term can be decomposed as

$$
N u(t)=\sum_{n=0}^{\infty} A_{n}
$$

where An are Adomian polynomials of $u_{0}, u_{1}, u_{2}, \ldots$ and it can be calculated by the formula

$$
A_{n}=\frac{1}{n !} \frac{d^{n}}{d \lambda^{n}}\left[N\left(\sum_{i=0}^{\infty} \lambda^{i} u_{i}\right)\right]_{\lambda=0}, n=0,1,2, \ldots
$$

Applying the operator $L^{-1}$ to both sides of (4) and substituting infinite series form of all equations into this equation we obtain

$$
\sum_{n=0}^{\infty} u_{n}(t)=G(t)-\left[R \sum_{n=0}^{\infty} u_{n}(t)+\sum_{n=0}^{\infty} A_{n}\right]
$$

where $G(t)$ is the term arising from the source term and the prescribed initial condition.

Finally, after selecting $u_{0}$ by using (8), we can start the iteration that will form the solution $u(t)=\sum_{n=0}^{\infty} u_{n}(t)$. This can be generalized to PDEs easily [4].

\section{TAYLOR MATRIX METHOD}

Taylor matrix method has been used to find the approximate solutions of partial differential, integral and integro-differential equations [24]-[26].This method shows the best advantage when the known functions in equation can be expanded to Taylor series about $x=c$ which converge rapidly. To get the best approximation to the accurate solution, we need to take more terms from the Taylor expansion of functions, in other words the truncation limit $N$ must be chosen large enough.

As in the previous section we will highlight only the points of the method that will be necessary for us. Details can be found in [27].

Considering the second order PDE

$$
a u_{x x}+b u_{t t}+c u_{x}+d u_{t}=g(x, t)
$$

With initial conditions

$$
u(x, 0)=f(x), u_{t}(x, 0)=h(x)
$$

We first assume that the solution is expressed in the form

$$
u(x, t)=\sum_{r=0}^{N} \sum_{s=0}^{N} a_{r, s}(x)^{r}(t)^{s}, a_{r, s}=\frac{1}{r ! s !} u^{(r, s)}(0,0)
$$

where $a_{r, s}$ are Taylor coefficients.

This solution function can be written in matrix form

$$
u(x, t)=X(x, t) A
$$

where $X(x, t)$ is $1 \times(N+1)^{2}$ matrix.

The relation between this matrix and its derivatives:

$$
\begin{aligned}
& u_{x}(x, t)=X(x, t) \bar{B} A \\
& u_{t}(x, t)=X(x, t) \tilde{B} A \\
& u_{x x}(x, t)=X(x, t)(\bar{B})^{2} A \\
& u_{t t}(x, t)=X(x, t)(\tilde{B})^{2} A
\end{aligned}
$$

where

$$
\begin{aligned}
& X(x, t)=\left[1 t t^{2} \cdots t^{N} x x t x t^{2} \cdots x t^{N} \cdots x^{N} x^{N} t \cdots x^{N} t^{N}\right]
\end{aligned}
$$

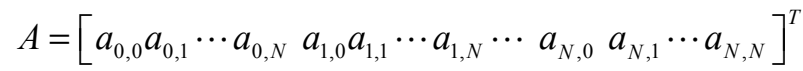

And

$$
B=\left[\begin{array}{ccccc}
0 & 1 & 0 & \cdots & 0 \\
0 & 0 & 2 & \cdots & 0 \\
\vdots & \vdots & \vdots & \cdots & \vdots \\
0 & 0 & 0 & \cdots & N \\
0 & 0 & 0 & \cdots & 0
\end{array}\right], \quad B=\left[\begin{array}{ccccc}
0 & I & 0 & \cdots & 0 \\
0 & 0 & 2 \mathrm{I} & \cdots & 0 \\
\vdots & \vdots & \vdots & \cdots & \vdots \\
0 & 0 & 0 & \cdots & N I \\
0 & 0 & 0 & \cdots & 0
\end{array}\right] \quad \tilde{B}=\left[\begin{array}{ccccc}
B & 0 & 0 & \cdots & 0 \\
0 & B & 0 & \cdots & 0 \\
\vdots & \vdots & \vdots & \cdots & \vdots \\
0 & 0 & 0 & \cdots & 0 \\
0 & 0 & 0 & \cdots & B
\end{array}\right]
$$

$I$ is the $(N+1) \times(N+1)$ identity matrix. 
Applying same procedure for $g(x, t)$, we can get:

$$
g(x, t)=X(x, t) G
$$

where

$$
g(x, t)=\sum_{r=0}^{N} \sum_{s=0}^{N} g_{r, s}(x)^{r}(t)^{s}, g_{r, s}=\frac{G^{(r, s)}(0,0)}{r ! s !}
$$

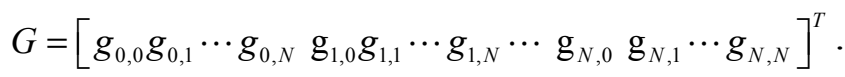

Substituting the expressions (12), (13) and (14) into (9) yields

$$
\left\{a(\bar{B})^{2}+b(\tilde{B})^{2}+c \bar{B}+d \tilde{B}\right\} A=G
$$

And it may be briefly written as

$$
W A=G
$$

For initial conditions we now consider new representation of $u(x, t)$ to make calculation easier:

$$
\begin{array}{r}
u(x, t)=X(x) Q(t) A \\
u_{t}(x, t)=X(x) Q(t) \tilde{B} A
\end{array}
$$

And

$$
\begin{gathered}
f(x)=X(x) F, F=\left[f_{0} f_{1} \cdots f_{N}\right]^{T}, f_{n}=\frac{f^{(n)}(0)}{n !} \\
h(x)=X(x) H, H=\left[h_{0} h_{1} \cdots h_{N}\right]^{T}, h_{n}=\frac{h^{(n)}(0)}{n !}
\end{gathered}
$$

where

$$
\begin{gathered}
X(x)=\left[\begin{array}{lllll}
1 & x & x^{2} & \cdots & x^{N}
\end{array}\right] \\
T(t)=\left[\begin{array}{lllll}
1 & t & t^{2} & \cdots & t^{N}
\end{array}\right] \\
Q(t)=\left[\begin{array}{ccccc}
T(t) & 0 & 0 & \cdots & 0 \\
0 & T(t) & 0 & \cdots & 0 \\
\vdots & \vdots & \vdots & \cdots & \vdots \\
0 & 0 & 0 & \cdots & 0 \\
0 & 0 & 0 & \cdots & T(t)
\end{array}\right] .
\end{gathered}
$$

By furnishing (16)-(19) into the (10) and by making some simplifications we get:

$$
\begin{aligned}
& S_{1}=Q(0) A=F \\
& S_{2}=Q(0) \tilde{B} A=H
\end{aligned}
$$

In order to provide higher accuracy, we form $\bar{W}$ and $\bar{G}$ by throwing away maximum number of row vectors from $W$ and $G$ so that the rank of the augmented matrix which is defined as

$$
[\tilde{W} ; \tilde{G}]=\left[\begin{array}{l}
S_{1} ; F \\
S_{2} ; H \\
\bar{W} ; \bar{G}
\end{array}\right]
$$

Cannot be smaller than $(N+1)^{2}$.

After applying Gauss elimination method to augmented matrix, we obtain $\tilde{\tilde{W}}$ and $\tilde{\tilde{G}}$ and Taylor coefficients are obtained as:

$$
A=(\tilde{\tilde{W}})^{-1} \tilde{\tilde{G}}
$$

\section{NUMERICAL EXAMPLES}

Let us now give the examples related with two methods for comparing the performance of them. We have used Maple 13 to handle with long difficult computations.

\section{A. Example 1}

Consider the linear inhomogeneous Klein-Gordon equation

$$
u_{t t}-u_{x x}+u=x\left(2+t^{2}\right)
$$

With Initial conditions

$$
u(x, 0)=0, u_{t}(x, 0)=0 .
$$

\section{1) Adomian decomposition method}

Operating with $L_{t}^{-1}=\int_{0}^{t} \int_{0}^{t}() d t d$.$t , Equation (21) becomes$

$$
\sum_{n=0}^{\infty} u_{n}(x, t)=x t^{2}+\frac{x t^{4}}{12}+L_{t}^{-1}\left[\left(\sum_{n=0}^{\infty} u_{n}\right)_{x x}\right]-L_{t}^{-1}\left[\sum_{n=0}^{\infty} u_{n}\right]
$$

We set the recursive relation as

$$
\begin{aligned}
& u_{0}(x, t)=x t^{2}+\frac{x t^{4}}{12} \\
& u_{k+1}(x, t)=L_{t}^{-1}\left(u_{k_{x x}}-u_{k}\right), k \geq 0
\end{aligned}
$$

that gives

$$
\begin{aligned}
& u_{0}(x, t)=x t^{2}+\frac{x t^{4}}{12} \\
& u_{1}(x, t)=L_{t}^{-1}\left(u_{0_{x x}}-u_{0}\right)=-\frac{x t^{4}}{12}-\frac{x t^{6}}{360} \\
& u_{2}(x, t)=L_{t}^{-1}\left(u_{1_{x x}}-u_{1}\right)=\frac{x t^{6}}{360}-\frac{x t^{8}}{20160}
\end{aligned}
$$

It is important to see here that the noise terms appear between the two components $u_{0}$ and $u_{1}$. The noise terms phenomenon [19] says that if the terms in $u_{0}$ are canceled by the terms in $u_{1}$, even though $u_{1}$ contains further terms, then the remaining non-canceled terms of $u_{0}$ may provide the exact solution of the problem. Therefore we may cancel the noise term $\frac{x t^{4}}{12}$ in $u_{0}$, so we get the exact solution as

$$
u(x, t)=x t^{2}
$$




\section{2) Taylor matrix method}

By taking $N=2$, we first assume that the problem has a solution in the form:

$$
u(x, t)=\sum_{r=0}^{2} \sum_{s=0}^{2} a_{r, s}(x)^{r}(t)^{s} .
$$

The fundamental matrix equation of this problem is

$$
\left\{(\tilde{B})^{2}+I-(\bar{B})^{2}\right\} A=W A=G .
$$

For $N=2$, we find

$$
[W ; G]=\left[\begin{array}{ccccccccc}
1 & 0 & 2 & 0 & 0 & 0 & -2 & 0 & 0 ; 0 \\
0 & 1 & 0 & 0 & 0 & 0 & 0 & -2 & 0 ; 0 \\
0 & 0 & 1 & 0 & 0 & 0 & 0 & 0 & -2 ; 0 \\
0 & 0 & 0 & 1 & 0 & 2 & 0 & 0 & 0 ; 2 \\
0 & 0 & 0 & 0 & 1 & 0 & 0 & 0 & 0 ; 0 \\
0 & 0 & 0 & 0 & 0 & 1 & 0 & 0 & 0 ; 1 \\
0 & 0 & 0 & 0 & 0 & 0 & 1 & 0 & 2 ; 0 \\
0 & 0 & 0 & 0 & 0 & 0 & 0 & 1 & 0 ; 0 \\
0 & 0 & 0 & 0 & 0 & 0 & 0 & 0 & 1 ; 0
\end{array}\right]
$$

Now using the initial conditions we have

$\left[S_{1} ; F\right]=\left[\begin{array}{ccccccccc}1 & 0 & 0 & 0 & 0 & 0 & 0 & 0 & 0 ; 0 \\ 0 & 1 & 0 & 0 & 0 & 0 & 0 & 0 & 0 ; 0 \\ 0 & 0 & 1 & 0 & 0 & 0 & 0 & 0 & 0 ; 0\end{array}\right]\left[S_{2} ; H\right]=\left[\begin{array}{ccccccccc}1 & 0 & 0 & 0 & 0 & 0 & 0 & 0 & 0 ; 0 \\ 0 & 0 & 0 & 0 & 1 & 0 & 0 & 0 & 0 ; 0 \\ 0 & 0 & 0 & 0 & 0 & 0 & 1 & 0 & 0 ; 0\end{array}\right]$

After removing the $k$ rows below from $[W ; G]$ to get $[\bar{W} ; \bar{G}]$ so that the rank of system $[\tilde{W} ; \tilde{G}]=\left[\begin{array}{l}S_{1} ; F \\ S_{2} ; H \\ \bar{W} ; \bar{G}\end{array}\right]$ can not be smaller than $(N+1)^{2}=9$, we have

$$
[\tilde{W} ; \tilde{G}]=\left[\begin{array}{cccccccccc}
1 & 0 & 0 & 0 & 0 & 0 & 0 & 0 & 0 & ; 0 \\
0 & 1 & 0 & 0 & 0 & 0 & 0 & 0 & 0 & 0 \\
0 & 0 & 1 & 0 & 0 & 0 & 0 & 0 & 0 & ; 0 \\
1 & 0 & 0 & 0 & 0 & 0 & 0 & 0 & 0 & ; 0 \\
0 & 0 & 0 & 0 & 1 & 0 & 0 & 0 & 0 & ; 0 \\
0 & 0 & 0 & 0 & 0 & 0 & 1 & 0 & 0 & 0 \\
1 & 0 & 2 & 0 & 0 & 0 & -2 & 0 & 0 & ; 0 \\
0 & 1 & 0 & 0 & 0 & 0 & 0 & -2 & 0 & 0 \\
0 & 0 & 1 & 0 & 0 & 0 & 0 & 0 & -2 & ; 0
\end{array}\right]
$$

And applying Gauss elimination process to this augmented matrix we have found the Taylor coefficients as:

$$
A=(\tilde{\tilde{W}})^{-1} \quad \tilde{\tilde{G}}=\left[\begin{array}{lllllllll}
0 & 0 & 0 & 0 & 0 & 1 & 0 & 0 & 0
\end{array}\right]^{T}
$$

Consequently, the exact solution is

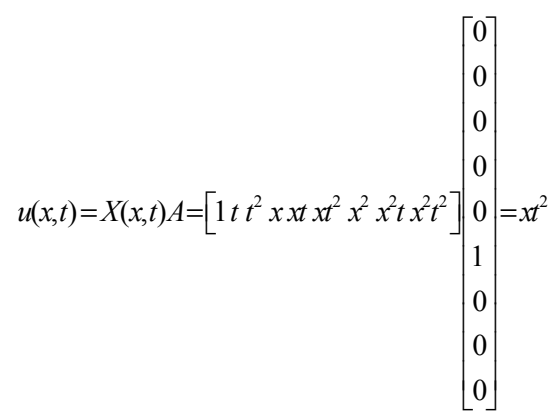

As in the ADM.

\section{B. Example 2}

Consider the Telegraph equation in [28]

$$
u_{t t}+u_{t}+u=u_{x x}
$$

Under the initial conditions

$$
u(x, 0)=e^{x}, u_{t}(x, 0)=-e^{x} \quad(0<x \leq 1, t \geq 0)
$$

\section{3) Adomian decomposition method}

Operating with $L_{t}^{-1}=\int_{0}^{t} \int_{0}^{t}() d t d$.$t on (29) and using the$ initial conditions:

$$
\begin{aligned}
& u(x, t)=u(x, 0)+t u_{t}(x, 0)+L_{t}^{-1}\left(u_{x x}-u_{t}-u\right) \\
& =e^{x}-t e^{-x}+L_{t}^{-1}\left(u_{x x}-u_{t}-u\right)
\end{aligned}
$$

And following the procedure presented before gives:

$$
\begin{aligned}
& \sum_{n=0}^{\infty} u_{n}(x, t)=e^{x}-t e^{x} \\
& +L_{t}^{-1}\left[\left(\sum_{n=0}^{\infty} u_{n}\right)_{x x}\right]-L_{t}^{-1}\left[\left(\sum_{n=0}^{\infty} u_{n}\right)_{t}\right]-L_{t}^{-1}\left[\sum_{n=0}^{\infty} u_{n}\right]
\end{aligned}
$$

Also writing recursive relation as:

$$
\begin{aligned}
& u_{0}(x, t)=e^{x}-t e^{x} \\
& u_{k+1}(x, t)=L_{t}^{-1}\left(u_{k_{x x}}-u_{k_{t}}-u_{k}\right), k \geq 0
\end{aligned}
$$

In view of (29) we obtain

$$
\begin{aligned}
& u_{1}(x, t)=L_{t}^{-1}\left(u_{0_{x x}}-u_{0_{t}}-u_{0}\right)=\frac{t^{2} e^{x}}{2 !} \\
& u_{2}(x, t)=L_{t}^{-1}\left(u_{1_{x x}}-u_{1_{t}}-u_{1}\right)=-\frac{t^{3} e^{x}}{3 !} \\
& u_{3}(x, t)=L_{t}^{-1}\left(u_{2_{x x}}-u_{2_{t}}-u_{2}\right)=-\frac{t^{4} e^{x}}{4 !}
\end{aligned}
$$$$
\text { : }
$$

Consequently, the solution can be predicted as:

$$
u(x, t)=e^{x}\left(1-t+\frac{t^{2}}{2 !}-\frac{t^{3}}{3 !}+\ldots\right)=e^{x} \cdot e^{-t}=e^{x-t}
$$

which is exact solution.

\section{4) Taylor matrix method}

Following the procedure in Section 3, the fundamental matrix equation is written as:

$$
\left\{(\tilde{B})^{2}+\tilde{B}+I-(\bar{B})^{2}\right\} A=W A=G
$$

where $\tilde{B}, \bar{B}$ and $I$ is $(N+1) \times(N+1)$ matrix . 
Proceeding for $N=9,11$ and 13 we obtain the approximate solution. A few terms of approximate solution are given as:

$$
\begin{aligned}
& u(x, t)=1-t+x-x t+\frac{x^{2}}{2}+\frac{t^{2}}{2}-\frac{x^{2} t}{2}+\frac{x^{2} t^{2}}{4}-\frac{x^{2} t^{3}}{12} \\
& +\frac{x^{3} t^{2}}{12}-\frac{x^{3} t^{3}}{36}+\frac{x^{2} t^{4}}{48}-\frac{x t^{6}}{720}+\frac{x t^{8}}{40320}+\frac{x t^{7}}{5040}-\frac{x t^{5}}{120} \\
& +\frac{x^{2} t^{10}}{7257600}+\frac{x^{2} t^{8}}{80640}-\frac{x^{2} t^{9}}{725760}-\frac{x^{4} t^{7}}{120960}+\frac{x^{5} t^{2}}{240}+ \\
& \frac{x^{5} t^{4}}{2880}-\frac{x^{6} t^{3}}{4320}-\frac{x^{8} t^{7}}{50803200}-\frac{x^{8} t}{40320}-\frac{x^{13}}{6227020800} \\
& -\frac{t^{13}}{6227020800}+\frac{x t^{11}}{39916800}-\frac{71 x^{12} t^{7}}{2414168064000} \\
& -\frac{x^{12} t^{8}}{275904921600}-\frac{x^{12} t^{12}}{458850656051200}-\frac{17 x^{13} t^{10}}{112983065395000} \\
& -\frac{x^{13} t^{11}}{1553517149184000}-\frac{x^{13} t^{13}}{387757880436326}
\end{aligned}
$$

Table I shows that the errors $L_{2}, L_{\infty}$ of $u(x, t)$ obtained by Taylor matrix for $N=11,13$.

Note that one can get the better approximation for greater $N$.

\section{Example 3}

Consider the Telegraph equation

$$
u_{t t}+6 u_{t}=9 u_{x x}
$$

Under the initial conditions

$$
u(x, 0)=\sin x, u_{t}(x, 0)=0 .
$$

\section{1) Adomian decomposition method}

Equation (37) can be written in operator as:

$$
L_{t} u(x, t)=9 L_{x} u(x, t)-6 \frac{\partial}{\partial t} u(x, t)
$$

where

$$
L_{t}=\frac{\partial^{2}}{\partial t^{2}}, L_{x}=\frac{\partial^{2}}{\partial x^{2}} .
$$

Applying the operator $L_{t}^{-1}=\int_{0}^{t} \int_{0}^{t}() d t d$.$t to the both sides of$ (37) we get

$$
u(x, t)=u(x, 0)+t u_{t}(x, 0)+L_{t}^{-1}\left(9 u_{x x}-6 u_{t}\right)
$$

And following the procedure presented before gives

$$
\sum_{n=0}^{\infty} u_{n}(x, t)=\sin x+9 L_{t}^{-1}\left[\left(\sum_{n=0}^{\infty} u_{n}\right)_{x x}\right]-6 L_{t}^{-1}\left[\left(\sum_{n=0}^{\infty} u_{n}\right)_{t}\right] .
$$

TABLE I: THE ERRORS $L_{2}, L_{\infty}$ FOR $N=11,13$

\begin{tabular}{|c|c|c|c|c|}
\hline$t$ & $\begin{array}{c}L_{2} \text {-error } \\
\text { for } N=11\end{array}$ & $\begin{array}{c}L_{2} \text {-error } \\
\text { for } N=13\end{array}$ & $\begin{array}{c}L_{\infty} \text {-error } \\
\text { for } N=11\end{array}$ & $\begin{array}{c}L_{\infty} \text {-error for } \\
N=13\end{array}$ \\
\hline 0.0 & $5.353555 \mathrm{E}-11$ & $1.261076 \mathrm{E}-11$ & $3.753458 \mathrm{E}-11$ & $1.228623 \mathrm{E}-11$ \\
\hline 0.1 & $6.943549 \mathrm{E}-11$ & $2.669647 \mathrm{E}-11$ & $6.852354 \mathrm{E}-11$ & $2.578625 \mathrm{E}-11$ \\
\hline 0.2 & $7.954889 \mathrm{E}-10$ & $1.034688 \mathrm{E}-10$ & $7.042345 \mathrm{E}-10$ & $9.899627 \mathrm{E}-11$ \\
\hline 0.3 & $9.857574 \mathrm{E}-10$ & $3.571254 \mathrm{E}-10$ & $9.883465 \mathrm{E}-10$ & $3.387709 \mathrm{E}-10$ \\
\hline 0.4 & $5.932033 \mathrm{E}-9$ & $1.078572 \mathrm{E}-9$ & $6.645354 \mathrm{E}-9$ & $1.014524 \mathrm{E}-9$ \\
\hline 0.5 & $6.043244 \mathrm{E}-9$ & $2.949374 \mathrm{E}-9$ & $7.223548 \mathrm{E}-9$ & $6.905494 \mathrm{E}-9$ \\
\hline 0.6 & $9.863054 \mathrm{E}-9$ & $7.474023 \mathrm{E}-9$ & $5.390324 \mathrm{E}-9$ & $1.632532 \mathrm{E}-9$ \\
\hline 0.7 & $3.990434 \mathrm{E}-8$ & $1.780584 \mathrm{E}-8$ & $8.508245 \mathrm{E}-8$ & $4.328865 \mathrm{E}-8$ \\
\hline 0.8 & $9.930425 \mathrm{E}-8$ & $8.303142 \mathrm{E}-8$ & $6.730634 \mathrm{E}-8$ & $1.254454 \mathrm{E}-8$ \\
\hline 0.9 & $5.770456 \mathrm{E}-8$ & $3.323374 \mathrm{E}-8$ & $5.850347 \mathrm{E}-8$ & $3.683875 \mathrm{E}-8$ \\
\hline 1.0 & $4.015345 \mathrm{E}-4$ & $1.897126 \mathrm{E}-4$ & $7.423408 \mathrm{E}-8$ & $1.607163 \mathrm{E}-8$ \\
\hline
\end{tabular}

Hence the approximate solution can be obtained as follows:

$u_{0}(x, t)=\sin x$

And

$$
u_{k+1}(x, t)=L_{t}^{-1}\left(9 u_{k_{x x}}-6 u_{k_{t}}\right), k \geq 0
$$

By (41) we have

$$
\begin{gathered}
u_{0}(x, t)=\sin x \\
u_{1}(x, t)=L_{t}^{-1}\left(9 u_{0_{x x}}-6 u_{0_{t}}\right)=-9\left(\frac{t^{2}}{2 !}\right) \sin x \\
u_{2}(x, t)=L_{t}^{-1}\left(9 u_{1_{x x}}-6 u_{1_{t}}\right)=81\left(\frac{t^{4}}{4 !}\right) \sin x+54\left(\frac{t^{3}}{3 !}\right) \sin x \\
u_{3}(x, t)=L_{t}^{-1}\left(9 u_{2_{x x}}-6 u_{2_{t}}\right) \\
=3^{6}\left(\frac{t^{6}}{6 !}\right) \sin x-12\left(3^{4}\right)\left(\frac{t^{5}}{5 !}\right) \sin x-4\left(3^{4}\right)\left(\frac{t^{4}}{4 !}\right) \sin x
\end{gathered}
$$

The solution in a series form is given by

$$
\begin{aligned}
& u(x, t)=\sin x\left(1-3 t+\frac{3^{2} t^{2}}{2 !}-\frac{3^{3} t^{3}}{3 !}+\frac{3^{4} t^{4}}{4 !}-\frac{3^{5} t^{5}}{5 !}\right)(1+3 t) \\
& =\sin x\left(e^{-3 t}\right)(1+3 t)
\end{aligned}
$$

which is the exact solution in closed form.

2) Taylor matrix method

Following the procedure in Section III, the fundamental matrix equation is written as:

$$
\left\{(\tilde{B})^{2}+6 \tilde{B}-(\bar{B})^{2}\right\} A=G
$$

where $(\tilde{B})^{2}, \tilde{B}$ and $(\bar{B})^{2}$ are all $(100) \times(100),(121) \times(121)$ and $(144) \times(144)$ matrices for $N=9,11$ and 13 respectively. 
TABLE II: ERROR ANALYSIS FOR $N=9,11$ AND 13

\begin{tabular}{|c|c|c|c|c|}
\hline$(x, t)$ & ADM=Exact & $N=9$ error & $N=11$ error & $N=13$ error \\
\hline$(0,0)$ & 0 & 0 & 0 & 0 \\
\hline$(0.1,0.1)$ & 0.0963063686 & $5.412 \mathrm{E}-10$ & $5.5405 \mathrm{E}-15$ & $8.174 \mathrm{E}-17$ \\
\hline$(0.2,0.2)$ & 0.1756197235 & $3.740 \mathrm{E}-10$ & $6.4303 \mathrm{E}-13$ & $4.092 \mathrm{E}-15$ \\
\hline$(0.3,0.3)$ & 0.2317447060 & $6.4234 \mathrm{E}-9$ & $8.1099 \mathrm{E}-10$ & $7.029 \mathrm{E}-14$ \\
\hline$(0.4,0.4)$ & 0.2650509066 & $4.3203 \mathrm{E}-8$ & $4.4988 \mathrm{E}-10$ & $4.649 \mathrm{E}-12$ \\
\hline$(0.5,0.5)$ & 0.2789127000 & $4.8938 \mathrm{E}-7$ & $5.9815 \mathrm{E}-9$ & $6.908 \mathrm{E}-11$ \\
\hline$(0.6,0.6)$ & 0.2777021322 & $7.5053 \mathrm{E}-7$ & $6.3123 \mathrm{E}-8$ & $5.595 \mathrm{E}-10$ \\
\hline$(0.7,0.7)$ & 0.2657304494 & $2.4530 \mathrm{E}-6$ & $4.8874 \mathrm{E}-7$ & $4.2734 \mathrm{E}-9$ \\
\hline$(0.8,0.8)$ & 0.2467528329 & $1.2303 \mathrm{E}-5$ & $8.5047 \mathrm{E}-7$ & $8.8852 \mathrm{E}-8$ \\
\hline$(0.9,0.9)$ & 0.2237943574 & $4.4032 \mathrm{E}-4$ & $5.7235 \mathrm{E}-6$ & $7.0398 \mathrm{E}-8$ \\
\hline$(1,1)$ & 0.1991482735 & $1.3032 \mathrm{E}-3$ & $4.6359 \mathrm{E}-5$ & $4.4952 \mathrm{E}-6$ \\
\hline
\end{tabular}

By taking $N=9,11$ and 13, we obtain the approximate solution. A few terms of approximate solution can be given as:

$$
\begin{aligned}
& u(x, t)=x-\frac{9 x t^{2}}{2}+\frac{3 x^{3} t^{2}}{4}-9 t^{3} x-\frac{x^{3}}{6}-\frac{3 x^{5} t^{2}}{80}-\frac{3 x^{3} t^{3}}{2}+\frac{27 x^{3} t^{4}}{32} \\
& -\frac{x^{5}}{120}+\frac{x^{7}}{5040}-\frac{x t^{5}}{120}+-\frac{27 x^{5} t^{5}}{4000}-\frac{729 x t^{8}}{380}+\frac{729 x t^{7}}{140}-\frac{x^{7} t^{2}}{580}- \\
& \frac{27 x^{5} t^{4}}{260}+\frac{27 x^{3} t^{6}}{32}+\frac{81 x t^{6}}{16}-\frac{x^{11} t^{5}}{2464000}-\frac{x^{9} t^{5}}{22400}-\frac{9 x^{7} t^{5}}{2800}+\cdots- \\
& \frac{14 x^{11} t^{12}}{88309760000}-\frac{27 x^{9} t^{12}}{401816000}-\frac{81 x^{9} t^{13}}{4404927380}-\frac{73 x^{11} t^{13}}{461407360000} .
\end{aligned}
$$

Errors are shown in Table II for comparison. As it can be easily seen, the results are healing by choosing the truncation limit $N$ greater.

\section{CONCLUSIONS}

We applied Adomian decomposition method and Taylor matrix method to solve some well known partial differential equations. It is observed that these methods can be an alternative way for the solution of partial differential equations that have no analytic solutions. In all examples we show that our methods have a good degree of accuracy. We give tables to clarify the importance of the choosing truncation limit $N$ sufficiently large.

Although the two methods provide good approximation, the decomposition method provides the fast convergent series of easily computable components and eliminates a lot of difficult computations required by Taylor matrix method. Consequently we can say that, Adomian decomposition method is more reliable and realistic than Taylor matrix method from the point of view of a practical implementation.

\section{REFERENCES}

[1] N. Bildik and A. Konuralp, "The use of variational iteration method, differential transform method and Adomian decomposition method for solving different types of nonlinear partial differential equations," International Journal of Nonlinear Sciences and Numerical Simulation, vol. 7, no. 1, pp. 65-70, 2006.

[2] E. V. Ferapontov and M. V. Pavlov, "Quasiclassical limit of coupled $\mathrm{KdV}$ equations. Riemann invariants and multi-Hamiltonian structure," Physica D: Nonlinear Phenomena, vol. 52, no. 2, pp. 211-219, 1991.
[3] S. T. M. Din et al., "Variational iteration method for re-formulated partial differential equations," International Journal of Nonlinear Sciences and Numerical Simulation, vol. 11, no. 2, pp. 87-92, 2010.

[4] A. M. Wazwaz, Partial Differential Equations: Methods and Applications, Taylor and Francis, 2002.

[5] P. D. Lax, "Development of singularities of solutions of nonlinear hyperbolic partial differential equations," Selected Papers Volume I, pp. 76-78, 2005.

[6] A. Brandt and I. Livshits, "Wave-ray multigrid method for standing wave equations," Electron. Trans. Numer. Anal., vol. 6, pp. 162-181, 1997.

[7] S. T. M. Din and M. A. Noor, "Solving Schrödinger equations by modified variational iteration method," World Applied Sciences Journal, vol. 5, no. 3, pp. 352-357, 2008.

[8] S. Momani and Z. Odibat, "Homotopy perturbation method for nonlinear partial differential equations of fractional order," Physics Letters A, vol. 365, no. 5, pp. 345-350, 2007.

[9] J. Shatah, "Normal forms and quadratic nonlinear Klein-Gordon equations," Communications on Pure and Applied Mathematics, vol. 38, no. 5, pp. 685-696, 1985.

[10] W. Strauss and L. Vazquez, "Numerical solution of a nonlinear Klein-Gordon equation," Journal of Computational Physics, vol. 28, no. 2, pp. 271-278, 1978.

[11] D. Noja, "Nonlinear Schrödinger equation on graphs: Recent results and open problems," Phil. Trans. R. Soc. A, vol. 372, no. 2007, 2014.

[12] H. Pecher, "Nonlinear small data scattering for the wave and Klein-Gordon equation," Mathematische Zeitschrift, vol. 185, no. 2, pp. 261-270, 1984.

[13] Z. Odibat and S. Momani, "A reliable treatment of homotopy perturbation method for Klein-Gordon equations," Physics Letters A, vol. 365 , no. 5 , pp. 351-357, 2007.

[14] A. S. V. R. Kanth and K. Aruna, "Differential transform method for solving the linear and nonlinear Klein-Gordon equation," Computer Physics Communications, vol. 180, no. 5, pp. 708-711, 2009.

[15] A. Mohebbi and M. Dehghan, "High order compact solution of the one-space-dimensional linear hyperbolic equation," Numerical Methods for Partial Differential Equations, vol. 24, no. 5, pp. 1222-1235, 2008.

[16] A. Saadatmandi and M. Dehghan, "Numerical solution of hyperbolic telegraph equation using the Chebyshev tau method," Numerical Methods for Partial Differential Equations, vol. 26, no. 1, pp. 239-252, 2010.

[17] S. A. Yousefi, "Legendre multiwavelet Galerkin method for solving the hyperbolic telegraph equation," Numerical Methods for Partial Differential Equations, vol. 26, no. 3, pp. 535-543, 2010.

[18] E. J. Parkes, B. R. Duffy, and P. C. Abbott, "The Jacobi elliptic-function method for finding periodic-wave solutions to nonlinear evolution equations," Physics Letters A, vol. 295, no. 5, pp. 280-286, 2002.

[19] G. Adomian, Solving Frontier Problems of Physics: The Decomposition Methoc, Kluwer Academic Publishers, 1994.

[20] G. Adomian and R. Rach, "Equality of partial solutions in the decomposition method for linear or nonlinear partial differential equations," Computers and Mathematics with Applications, vol. 19, no. 12, pp. 9-12, 1990. 
[21] D. J. Evans and K. R. Raslan, "The Adomian decomposition method for solving delay differential equation," International Journal of Computer Mathematics, vol. 82, no. 1, pp. 49-54, 2005.

[22] A. -M. Wazwaz, "Adomian decomposition method for a reliable treatment of the Bratu-type equations," Applied Mathematics and Computation, vol. 166, no. 3, pp. 652-663, 2005.

[23] A. -M. Wazwaz, "A reliable modification of Adomian decomposition method," Applied Mathematics and Computation, vol. 102, no. 1, pp. 77-86, 1999.

[24] B. Bülbül and M. Sezer, "A Taylor matrix method for the solution of a two-dimensional linear hyperbolic equation," Applied Mathematics Letters, vol. 24, no. 10, pp. 1716-1720, 2011.

[25] B. Bülbül and M. Sezer, "Numerical solution of Duffing equation by using an improved Taylor matrix method," Journal of Applied Mathematics, vol. 2013, 2013.

[26] S. Yalçinbaş and M. Sezer, "The approximate solution of high-order linear Volterra-Fredholm integro-differential equations in terms of Taylor polynomials," Applied Mathematics and Computation, vol. 112, no. 2-3, pp. 291-308, 2000.

[27] B. Bülbül and M. Sezer, "Taylor polynomial solution of hyperbolic type partial differential equations with constant coefficients," International Journal of Computer Mathematics, vol. 88, no. 3, pp. 533-544, 2011.

[28] D. J. Evans and H. Bulut, "The numerical solution of the telegraph equation by the alternating group explicit (AGE) method," International Journal of Computer Mathematics, vol. 80, no. 10, pp. 1289-1297, 2003.

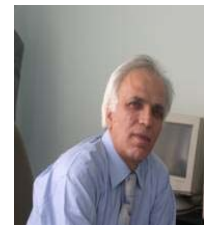

Necdet Bildik was born in Sivas/Turkey in 1951. He graduated from Ankara University in 1974. He earned the M.Sc. degree in University of Louisville, Kentucky, USA in 1978. He was awarded the Ph.D. degree in Oklahoma State University, USA in 1982. He was an assistant professor in 1988 and also he became an associate professor in 1995. He was promoted to be professor in 2003. He is interested in numerical analysis, ordinary, partial and non-linear differential equations, ergodic theory, stability theory.

$\mathrm{He}$ has over than a hundred published articles in the national and international journals and conferences. He also serves as a reviewer for many international journals.

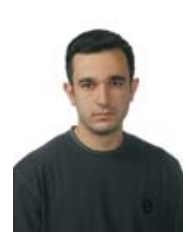

Sinan Deniz is a research assistant of mathematics in Celal Bayar University. He obtained his B.Sc degree from the Department of Mathematics at Fatih University, İstanbul/Turkey in 2012 and he is currently studying for M.Sc. degree at Celal Bayar University, Manisa/Turkey. He works on the systems of delay differential equations and numerical solutions of nonlinear differential equations, analytical methods for partial differential

\section{equations.}

